\title{
Pediatric Bell's palsy: prognostic factors and treatment outcomes
}

\author{
Abdulhalim Aysel ${ }^{1 \oplus}$, Togay Müderris ${ }^{2 \oplus}$, Fatih Yılmaz $^{1 \oplus}$, Taşkın Tokat ${ }^{1 \oplus}$, \\ Aynur Aliyeva $^{1 \oplus}$, Özgür Özdemir Şimşek ${ }^{3 \oplus}$, Enver Altaş ${ }^{1 \oplus}$ \\ ${ }^{1}$ Department of Otorhinolaryngology, Head and Neck Surgery, Bozyaka Training and Research Hospital, University of Health Sciences, \\ Izmir; ${ }^{2}$ Department of Otorhinolaryngology and Head and Neck Surgery, Bakırçay University Izmir Çiğli Training and Research \\ Hospital, Izmir; ${ }^{3}$ Dr. Behçet Uz Children's Training and Research Hospital, University of Health Sciences, İzmir, Turkey.
}

\begin{abstract}
Background. Idiopathic facial paralysis or Bell's palsy is the most common type of peripheral facial paralysis. Children with Bell's palsy is an uneasy situation for the family and physician with questions about the etiology, treatment options and the healing process. Here, we aimed to compare the epidemiologic features and prognostic factors of patients with Bell's palsy aged $<18$ years.
\end{abstract}

Methods. Records of patients with Bell's palsy who were admitted to our clinic between January 2008 and December 2017 were evaluated.

Results. Forty-seven patients with Bell's palsy were included to this study. The patients' ages varied between 7 and $17(14.7 \pm 2.5)$ years. At the end of at least 6 months of follow-up, $32(68.1 \%)$ of the patients presented with House Brackmann (HB) grade 1 facial paralysis, while 12 (25.5\%) of them had grade 2 and $3(6.4 \%)$ of them had grade 3 facial paralysis. Mean neutrophil-to-lymphocyte ratio (NLR) in patients with advanced grades (grade 4, $5,6)$ was higher, compared to that of patients with grade 2 and $3(4.10 \pm 1.06$ vs $1.34 \pm 1.02(\mathrm{p}<0.001)$.

Conclusions. In our study, the response rate to treatment was high. In differential diagnosis, congenital anomalies, malignancy, trauma, middle ear infection and surgery should be considered. In addition, NLR at admission can be considered as a prognostic factor.

Key words: Bell palsy, children, inflammation, neutrophils, neutrophil-to-lymphocyte ratio, prognosis.

Acute idiopathic peripheral facial paralysis (PFP) or Bell's palsy (BP), can be seen in all age groups. ${ }^{1}$ In general, its frequency is considered to be $20-30 / 100,000 .{ }^{1}$ It is also the most common cause of unilateral facial paralysis and constitutes $60-75 \%$ of paralysis of the facial nerve., ${ }^{2,3}$ Nine percent of the patients have a previous history of paralysis. ${ }^{1}$ Bilateral paralysis is observed in $0.3 \%$ of the patients. ${ }^{1}$ Although the incidence of BP in childhood is

Abdulhalim Aysel

ahalimaysel@gmail.com

Received 1st October 2019, revised 10th December 2019, 24th February 2020, accepted 11th May 2020.

This study was presented as an oral presentation at the 40th National Congress of Otolaryngology and Head and Neck Surgery, November 7-11 2018, Antalya, Turkey. not known clearly, Peitersen reported that it is seen in $14 \%$ of the patients under 15 years of age, among $2500 \mathrm{BP}$ patients. ${ }^{4} \mathrm{BP}$ prognosis is usually good and over $85 \%$ of patients have a significant improvement in 3-4 weeks. ${ }^{4}$

Genetic factors, infection and autoimmune diseases that cause vascular ischemia and inflammation, temporal bone fractures, neoplastic lesions can be observed in the ethiology. ${ }^{4}$ Speech and social communication may be affected due to the weakness of facial muscles; most importantly, eye and vision may deteriorate due to decreased corneal reflex. ${ }^{5}$

Bell's palsy in children is an uneasy situation for the family and physician with questions about the etiology, treatment options and the healing 
process. ${ }^{6}$ In these patients, history taking and neuro-clinical examination should be performed carefully; and audiological evaluation, biochemical analysis and radiological imaging methods should be followed if possible. ${ }^{6}$ These evaluations are important to reveal the possible cause of paralysis. ${ }^{6}$ Although there is no universally accepted treatment modality for the treatment of BP in children, corticosteroids, antiviral agents and decompression surgery seem to be the best alternatives at the moment. ${ }^{6}$ Corticosteroids are the most commonly used agents. ${ }^{6}$ In particular, initiation of corticosteroid therapy in the first week of the disease improves muscle function and decreases complication rates. $^{6}$ Although antiviral agents are useful in the herpes zoster facial paralysis, its usefulness is limited in idiopathic facial paralysis. ${ }^{6,7}$ Surgical treatment may be considered in cases where there is no clinical improvement or no regeneration is observed with electrophysiological tests, although with controversial results. ${ }^{6,7}$

Bell's palsy is the most common cause of PFP in childhood, and proper management of $\mathrm{BP}$ is essential for pediatricians, otolaryngologists and general practitioners since it can be a devastating situation for the family. 6,7 In this study, epidemiological characteristics, treatment responses and possible prognostic factors of pediatric patients diagnosed with BP were investigated.

\section{Material and Methods}

\section{Study design and patients}

We evaluated the records of patients aged $<18$ years who received treatment for PFP at our clinic between January 2008 and December 2017, with a minimum follow-up period of 6 months. The institutional University of Health Sciences, İzmir Bozyaka Training and Research Hospital review board approved this retrospective study (04/17.10.2018).

Age, sex, duration from the onset to the treatment, previous history of facial palsy, such as pain and skin eruption around the affected ear, hyperacusis, upper respiratory tract infection history, family history of facial paralysis, neurootologic examination and laboratory tests (complete blood count, serum biochemistry panel, serologic tests for herpes simplex virus-1 [HSV], varicella zoster virus [VZV], mumps virus and Mycoplasma pneumoniae), radiologic tests such as brain magnetic resonance and/or computed tomography, and treatment results of all patients were evaluated. Peripheral facial paralysis without an apparent specific etiology was considered as BP. During the first consultation and evaluation of treatment outcomes, the House-Brackmann (HB) facial nerve grading system was used to identify the degree of paralysis (Table I). ${ }^{8}$ In patients diagnosed with progressive facial paralysis, only the last grade of facial paralysis was taken into consideration. Moreover, all patients were

Table I. House Brackman facial palsy grading system.

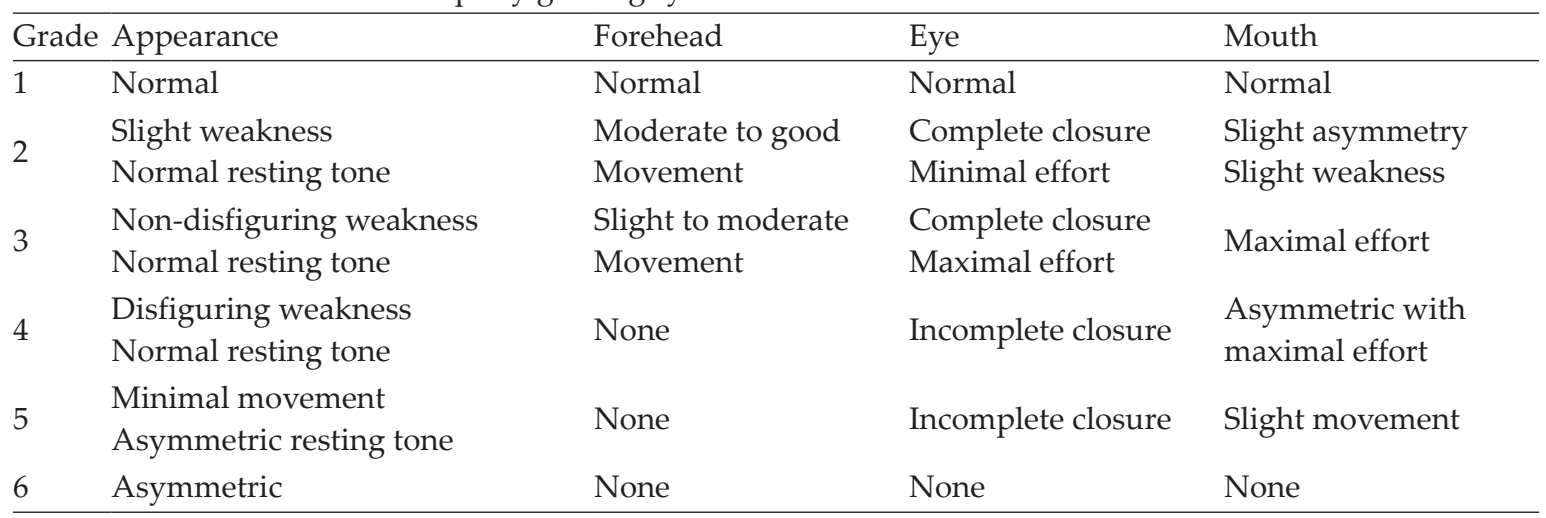


assessed by an ophthalmologist and physical therapist for designing a suitable physical therapy program that included facial muscle training. All patients underwent an initial blood test prior to treatment, and the neutrophilto-lymphocyte ratio (NLR), thrombocyte-tolymphocyte ratio (TLR) and mean platelet volume (MPV) levels of all patients were examined. At the 6th month follow-up, HB grade 1 was considered as "complete" recovery and HB grade 2 as "almost complete" recovery. If the patients had a facial paralysis $\mathrm{HB}$ grade 3 or over at the last follow up visit, they were considered as "partial" recovery if they showed an improvement in terms of HB grading; and "no recovery" if there was no improvement.

\section{Treatment protocols}

At our clinic, the treatment for patients with BP includes oral or intravenous methyl prednisolone $(1 \mathrm{mg} / \mathrm{kg} /$ day) at tapered doses for 14 days. Further, antiviral therapy was included for the treatment of patients with a positive recent upper respiratory tract infection history or positive serologic test results for aforementioned viruses.

\section{Statistical analysis}

Descriptive statistics (arithmetic mean, median, minimum, maximum, standard deviation, and standard error) were first calculated on the basis of the obtained data. Spearman's rho correlation analysis, Kruskal-Wallis test, and Mann-Whitney $U$-test were performed in accordance with the data distribution. $\mathrm{p}<0.05$ was considered statistically significant. The Statistical Package for the Social Sciences (SPSS) software was used for statistical analysis.

\section{Results}

\section{Patient characteristics}

Of the 51 patients with PFP, 4 (7.8\%) patients with different etiologic factors were excluded. Of these excluded patients, two was secondary to acute otitis media, one had acoustic neuroma and one patient had osteopetrosis. The remaining 47 patients with $\mathrm{PFP}$ were included to the study with the diagnosis of BP.

Period from the onset of the disease to hospital admission ranged from 1 to 10 days (mean, $3.48 \pm 0.21)$ days. Three $(6.3 \%)$ patients were diagnosed with progressive facial paralysis while five $(10.6 \%)$ patients had a positive family history. Nine (19.1\%) patients had a history of upper respiratory tract infection, accordingly received antiviral therapy.

Three $(6.3 \%)$ cases of recurrent facial paralysis were observed. The patients' ages ranged from 7 to 17 (mean, $14.7 \pm 2.5$ ) years. Twenty-three (48.9\%) of the patients were boys and $24(51.1 \%)$ of them were girls. The follow-up period ranged from 6 to 108 months (mean, $61.23 \pm 31.83$ ). Paralysis was observed on the right side in 23 of the patients (48.9\%); meanwhile, $51.1 \%$ ( $n=$ 24 ) of the patients experienced paralysis on the left side.

HSV-1 DNA was detected in two of nine patients with a history of upper respiratory tract infection and mycoplasma Ig M serology was positive in one patient.

When facial paralysis was investigated in terms of seasonal distribution, 17 (36.2\%), 12 (25.5\%), $12(25.5 \%)$, and $6(12.8 \%)$ patients presented with paralysis during the spring, winter, summer, and fall, respectively.

Patients' initial and last facial paralysis grades are summarized in Table II. Overall, $93.6 \%$ of the children had complete or almost complete recovery, with the rest of them showed partial improvement. No association was found between gender, side of the paralysis and season of the paralysis and pre-treatment and post-treatment grades ( $>00.05)$. Cranial and/ or temporal magnetic resonance examination of 6 patients who did not exhibit complete recovery after 3 weeks of treatment revealed no pathologic lesion. 
Table II. Clinical grades of patients $(\mathrm{N}=47)$ according to House Brackman facial palsy grading system.

\begin{tabular}{ccc}
\hline Clinical grades & Frequency $(\mathrm{N})$ & Percent \\
\hline Initial & 1 & \\
2 & 21 & 2.1 \\
3 & 12 & 24.7 \\
4 & 10 & 21.3 \\
5 & 3 & 6.4 \\
6 & & \\
Follow-up & 32 & 68.1 \\
1 & 12 & 25.5 \\
2 & 3 & 6.4 \\
3
\end{tabular}

\section{Laboratory results}

The mean NLR, TLR and MPV at initial admission were $2.69 \pm 1.57,147.33 \pm 76.38$, and $9.01 \pm 1.71 \mathrm{fL}$, respectively. Mean NLR in patients with advanced grades (grade 4, 5, 6) was higher $(4.10 \pm 1.06)$, compared to that of patients with grade 2 and grade $3(1.34 \pm 1.02)$; $\mathrm{p}<0.001$ (Fig. 1). No statistically significant difference was found for TLR and MPV ( $p=$ $0.146, p=648$, respectively).

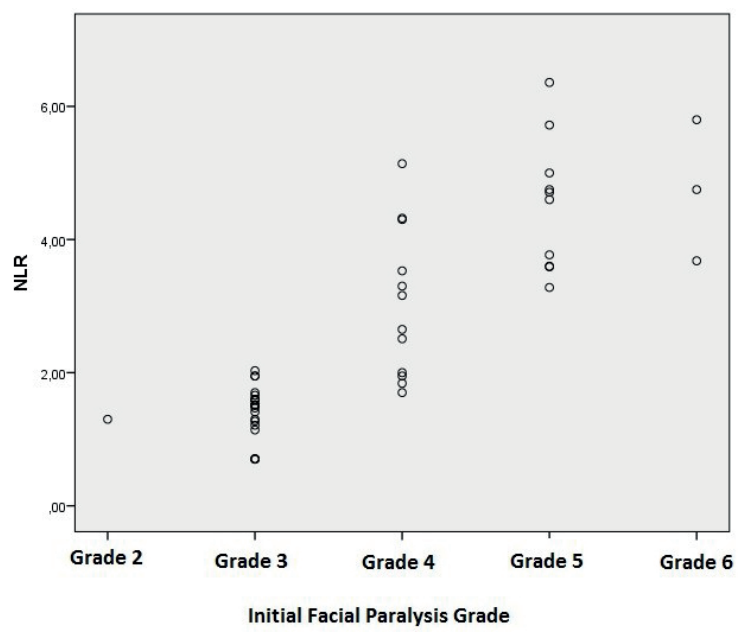

Fig. 1. Neutrophil/lymphocyte ratio (NLR) according to initial facial paralysis grade (House Brackmann). Patients with advanced grades (grade 4, 5, 6) had higher $(4.10 \pm 1.06)$ NLR, compared to that of patients with grade 2 and grade $3(1.34 \pm 1.02) ; \mathrm{p}<0.001$

\section{Discussion}

$\mathrm{BP}$ is the most common cause of unilateral facial paralysis and accounts for $60-70 \%$ of all facial nerve paralysis cases. ${ }^{9}$ Likewise, $\mathrm{BP}$ is the most common cause of PFP in childhood, and proper management is essential for the medical professionals since parents of the patients are usually panicky and demanding. ${ }^{9}$

The prognosis of BP is extremely good, more than $85 \%$ of all patients exhibit complete/near complete recovery in 3 to 4 weeks. ${ }^{9}$ Treatment is usually based on steroids and antiviral agents and surgical treatment is generally kept for rare cases where no regeneration based on electro physiologic tests or if no clinical recovery is observed. ${ }^{9}$

Cha et al. ${ }^{9}$ reported that BP was the most common condition in childhood facial paralysis $(66.7 \%)$ and cases were mostly (62.5\%) presenting with HB grade 5 facial paralysis. At the end of the 6-month follow-up, facial paralysis regressed to $\mathrm{HB}$ grade 1 or 2 in $91.6 \%$ of the patients. Similarly, May et al. ${ }^{10}$ reported 170 patients under 18 years of age with PFP, and $\mathrm{BP}$ was the most common cause $(42 \%)$, followed by trauma (21\%), infection (13\%), congenital causes $(8 \%)$ and malignancy $(2 \%)$. In other studies, the incidence of PFP secondary to acute otitis media was reported between $4 \%$ and $37 \%$ and the incidence of tumor-induced PFP was between $2 \%$ and $12 \% .{ }^{11-13}$

$\mathrm{BP}$ was the cause of peripheral facial paralysis in 93.1\% of the patients in the present study. This higher BP rate in patients with peripheral facial paralysis compared to previous studies may be attributed to easier access of the families to healthcare services, hence early diagnosis and proper management of acute otitis media and other possible causes in children in recent years.

Although recurrent facial paralysis is rare in children, the incidence of recurrent PFP has been reported in $3-15 \%$ of studies. ${ }^{11-14}$ In our study, 3 patients $(6.3 \%)$ had recurrent BP with two patients had two facial paralysis attacks and one patient had three facial paralysis attacks. 
The family history of all these three patients was positive. In the literature, the family history of $\mathrm{BP}$ varies between $8.5 \%$ and $10.3 \%,{ }^{15,16}$ which was also positive in five $(10.6 \%)$ patients in our series. It is essential to have a detailed family history in patients with PFP, since the recurrence of paralysis is significantly higher in patients with family history.

Facial paralysis usually onsets suddenly, but it can be seen progressively in some cases. ${ }^{16}$ Kasse et al. ${ }^{17}$ reported the rate of sudden onset facial paralysis as $72.5 \%$ while it was progressive in $27.5 \%$ of the patients in their study with 1521 BP patients in all age groups. They reported that the progressive course was associated with significantly worse prognosis. ${ }^{17}$ In our study population, progressive facial paralysis was detected in three $(6.3 \%)$ patients and complete recovery was observed in the 6th month in all these patients. Although patient numbers are limited, pediatric patients with progressive paralysis seem to heal better.

We did not observe any bilateral cases in our series, accordingly bilateral BP is very rare in the literature. ${ }^{1,17,18}$ Most studies did not show any gender differences among children with $\mathrm{PFP}^{18-21}$ as in our study, in which no gender difference was found both in incidence and treatment responses. It has been suggested that, there might be a seasonal distribution of the paralysis with different results. While Peitersen et al. ${ }^{4}$ did not indicate a seasonal difference, Devriese et al. ${ }^{18}$ reported that BP was more common in winter. We did not find a significant difference in seasonal distribution of pediatric patients with PFP ( $p>0.05)$.

Dhiravibulya $^{19}$ reported a complete recovery (HB grade 1 ) rate $(61.7 \%)$ and a nearly complete recovery rate ( $\mathrm{HB}$ grade 2$)(38.2 \%)$ in 39 pediatric patients at the end of 7 months. Chen and Wong reported that all cases except for one $(3.1 \%)$ patient were fully cured (completeHB grade 1; almost complete-HB grade 2) and complete recovery rate in the first 3 weeks was $68.8 \%$ in their study. ${ }^{20}$ In our study, complete recovery was achieved in 32 patients (68.1\%), almost complete recovery was achieved in 12 patients $(25.5 \%)$ at the end of 6 months of followup, which are similar to the previous studies. These high recovery rates emphasize the importance of proper treatment, since sequalae of PFP might be psychologically catastrophic to children and their parents, even though it is not a life threatening disease.

Epstein-Barr virus, cytomegalovirus, mumps virus, rubella virus, varicella zoster virus, coxsackie virus, M. pneumoniae and herpes simplex virus can play a role in the etiology of PFP. ${ }^{20,21}$ Kang et al. ${ }^{22}$ found that the combined treatment with acyclovir and prednisolone in advanced grade (HB grade 5, 6) patients was higher than that of prednisolone alone. In our study, HSV-1 DNA was detected in 2 of 9 patients with a history of upper respiratory tract infection and M. pneumoniae Ig M serology was positive in 1 patient. Patients with herpes simplex were treated with acyclovir plus steroid treatment, patient with mycoplasma for 10 days with standard steroid plus clarithromycin treatment in patients with after the treatment, complete improvement was observed in all 3 patients.

Corticosteroids are still the most commonly used agents in the treatment of PFP. ${ }^{23,24}$ Sullivan et al. reported a high recovery rate of $90 \%$ with prednisolone monotherapy in $\mathrm{BP} .{ }^{23}$ It is a difficult clinical decision to discontinue a childhood case without treatment even in low grade paralysis that could be usually followedup without any treatment in adults, and it should be taken into consideration that lack of treatment will have a lasting effect on the life of the child..$^{25}$ Although steroid related side effects can be used as a data against steroid use, none of the patients in our study had any apparent adverse effects of steroid therapy in therapeutic doses. ${ }^{25}$ We therefore suggest steroid therapy to all pediatric patients with BP. An MRI scan should also be performed if there is no response to proper treatment for more than 3 weeks, in cases with recurrent PFP, in children with additional neurological abnormalities and when there is a suspicion of malignancy. ${ }^{26}$ 
In our study, three patients with progressive facial paralysis showed no pathology in cranial MRI and CT examinations; however, a 16-year-old boy with HB grade 3 PFP who had no improvement after 2 weeks of treatment was found to have osteopetrosis in the cranial $\mathrm{CT}$ and MRI examinations. No improvement in 6th month control was observed in this patient. A 15-year-old boy with $\mathrm{HB}$ grade 4 left PFP who had not recovered after 3 weeks of standard treatment was found to have a soft tissue consistent with Schwannoma, measured approximately $5 \times 3 \mathrm{~mm}$ in the left internal acoustic canal in the MRI. Complete recovery was achieved in the follow-up controls. No increase in lesion size was observed in the control MRI's that were performed annually.

Complications such as synkinesis, or keratitis can be seen after BP. ${ }^{27}$ We observed synkinesis in 2 patients $(4.2 \%)$ and hemifacial spasm in 1 patient $(2.1 \%)$. Although these are rare complications, patients have to be followed up closely for a couple of years even if complete recovery is achieved.

Several recent studies have utilized the NLR and TLR as inflammation markers. ${ }^{28,29}$ Reasonably, a high NLR was reported among patients with BP. ${ }^{28,29}$ Ozler et al. ${ }^{30}$ reported considerably high levels of NLR in patients who did not recover from facial paralysis within 3 months of treatment follow-up. Moreover, a correlation was found between the grades of facial paralysis and NLR upon admission. ${ }^{30}$ We observed significantly high rates of NLR in patients with a high onset grade of facial paralysis; however, no significant correlation was observed between the MPV, TLR, and FBG levels and facial paralysis degree. We also found that higher NLR rates were associated with poor prognosis. NLR can be suggested as an inflammatory marker that may predict the prognosis of facial paralysis in children, even in the early periods of the paralysis.

$\mathrm{BP}$ in children is a relatively rare situation compared to adults and causes significant concerns in doctors and parents due to functional and aesthetic concerns. In differential diagnosis, congenital anomalies, malignancy, trauma, middle ear infection and surgery should be considered. High NLR can be used as poor prognostic marker in BP. Steroid treatment to all patients and antiviral agents in selected cases promise excellent results.

\section{REFERENCES}

1. Zandian A, Osiro S, Hudson R, et al. The neurologist's dilemma: a comprehensive clinical review of Bell's palsy, with emphasis on current management trends. Med Sci Monit 2014; 20: 83-90.

2. Taverner D, Kemble F, Cohen SB. Prognosis and treatment of idiopathic facial (Bell's) palsy. Br Med J 1967; 4: 581-582.

3. Baugh RF, Basura GJ, Ishii LE, et al. Clinical practice guideline: Bell's palsy. Otolaryngol Head Neck Surg 2013; 149 (Suppl 3): S1-S27.

4. Peitersen E. Bell's palsy: the spontaneous course of 2,500 peripheral facial nerve palsies of different etiologies. Acta Otolaryngol Suppl 2002; 549: 4-30.

5. Tiemstra JD, Khatkhate N. Bell's palsy: diagnosis and management. Am Fam Physician 2007; 76: 9971002.

6. Stew B, Williams H. Modern management of facial palsy: a review of current literature. Br J Gen Pract 2013; 63: 109-110.

7. Goudakos JK, Markou KD. Corticosteroids vs corticosteroids plus antiviral agents in the treatment of Bell palsy: a systematic review and meta-analysis. Arch Otolaryngol Head Neck Surg 2009; 135: 558564.

8. House JW, Brackmann DE. Facial nerve grading system. Otolaryngol Head Neck Surg 1985; 93: 146147.

9. Cha CI, Hong CK, Park MS, Yeo SG. Comparison of facial nerve paralysis in adults and children. Yonsei Med J 2008; 49: 725-734.

10. May M, Klein SR. Differential diagnosis of facial nerve palsy. Otolaryngol Clin North Am 1991; 24: 613-645.

11. Özkale Y, Erol İ, Sayg1 S, Yılmaz İ. Overview of pediatric peripheral facial nerve paralysis: analysis of 40 patients. J Child Neurol 2015; 30: 193-199.

12. Shih WH, Tseng FY, Yeh TH, Hsu CJ, Chen YS. Outcomes of facial palsy in children. Acta Otolaryngol 2009; 129: 915-920. 
13. Evans AK, Licameli G, Brietzke S, Whittemore K, Kenna M. Pediatric facial nerve paralysis: patients, management and outcomes. Int J Pediatr Otorhinolaryngol 2005; 69: 1521-1528.

14. Eidlitz-Markus T, Gilai A, Mimouni M, Shuper A. Recurrent facial nerve palsy in paediatric patients. Eur J Pediatr 2001; 160: 659-663.

15. Lunan R, Nagarajan L. Bell'spalsy: a guideline proposal following a review of practice. J Paediatr Child Health 2008; 44: 219-220.

16. Stew B, Williams H. Modern management of facial palsy: a review of current literature. Br J Gen Pract 2013; 63: 109-110.

17. Kasse CA, Cruz OL, Leonhardt FD, Testa JR, Ferri RG, Viertler EY. The value of prognostic clinical data in Bell's palsy. Braz J Otorhinolaryngol 2005; 71: 454458.

18. Devriese PP, Schumacher T, Scheide A, de Jongh RH, Houtkooper JM. Incidence, prognosis and recovery of Bell's palsy. A survey of about 1000 patients (19741983). Clin Otolaryngol Allied Sci 1990; 15: 15-27.

19. Dhiravibulya K. Outcome of Bell's palsy in children. J Med Assoc Thai 2002; 85: 334-339.

20. Chen WX, Wong V. Prognosis of Bell's palsy in children-analysis of 29 cases. Brain Dev 2005; 27: 504-508.

21. Ogita S, Terada K, Niizuma T, Kosaka Y, Kataoka N. Characteristics of facial nerve palsy during childhood in Japan: frequency of varicella zoster virus association. Pediatr Int 2006; 48: 245-249.
22. Kang HM, Jung SY, Byun JY, Park MS, Yeo SG. Steroid plus antiviral treatment for Bell's palsy. J Intern Med 2015; 277: 532-539.

23. Sullivan FM, Swan IR, Donnan PT, et al. Early treatment with prednisolone or acyclovir in Bell's palsy. N Engl J Med 2007; 357: 1598-1607.

24. Pitaro J, Waissbluth S, Daniel SJ. Do children with Bell's palsy benefit from steroid treatment? A systematic review. Int J Pediatr Otorhinolaryngol 2012; 76: 921-926.

25. Pavlou E, Gkampeta A, Arampatzi M. Facial nerve palsy in childhood. Brain Dev 2011; 33: 644-650.

26. Veillon F, Taboada LR, Eid MA, et al. Pathology of the facial nerve. Neuroimaging Clin N Am 2008; 18: 309-320.

27. Holland NJ, Weiner GM. Recent developments in Bell's palsy. BMJ 2004; 329: 553-557.

28. Eryilmaz A, Basal Y, Tosun A, Kurt Omurlu I, Basak S. The neutrophil to lymphocyte ratios of our pediatric patients with Bell's palsy. Int J Pediatr Otorhinolaryngol 2015; 79: 2374-2377.

29. Kiliçkaya MM, Tuz M, Yariktaş M, Yasan H, Aynalı G, Bagci Ö. The importance of the neutrophillymphocyte ratio in patients with idiopathic peripheral facial palsy. Int J Otolaryngol 2015; 2015: 981950.

30. Ozler GS, Gunak G. Neutrophil-lymphocyte ratio: a new predictive and prognostic factor in patients with Bell palsy. J Craniofac Surg 2014; 25: 944-945. 